\title{
Performance Evaluation of Content-Based Image Retrieval on Feature Optimization and Selection Using Swarm Intelligence
}

\author{
Kirti Jain ${ }^{1}$, \\ ${ }^{1}$ (Computer Science \&Engg Dept., LNCT GROUP \\ BHOPAL, INDIA)
}

\author{
Dr.Sarita Singh Bhadauria ${ }^{2}$ \\ ${ }^{2}$ (ElectronicsDept.,M.I.T.S. \\ GWALIOR, INDIA)
}

\begin{abstract}
The diversity and applicability of swarm intelligence is increasing everyday in the fields of science and engineering. Swarm intelligence gives the features of the dynamic features optimization concept. We have used swarm intelligence for the process of feature optimization and feature selection for content-based image retrieval. The performance of content-based image retrieval faced the problem of precision and recall. The value of precision and recall depends on the retrieval capacity of the image. The basic raw image content has visual features such as color, texture, shape and size. The partial feature extraction technique is based on geometric invariant function. Three swarm intelligence algorithms were used for the optimization of features: ant colony optimization, particle swarm optimization (PSO), and glowworm optimization algorithm. Coral image dataset and MatLab software were used for evaluating performance.
\end{abstract}

Keywords-CBIR; Swarm intelligence; feature extraction;SIFT transform; GSO(glowwarm swarm optimization)

\section{INTRODUCTION}

Content-based image retrieval has started playing a major role in multimedia data storage and retrieval. A large amount of multimedia data is generated in today's times, such as image and video carton. For the purpose of online retrieval of these data, content-based image retrieval is used. The major issue in this process is precision and recall of retrieval image according to the query image [1,2]. For improvement of precision and recall, various authors used feature optimization and feature selection technique. The feature selection and feature optimization technique improved the performance of content-based image retrieval. For the extraction of feature, various pixel-based techniques and transform-based techniques were used. For transform-based technique, Wavelet transform function, Gabor transform function, SIFT transform function, and many other transform function-based techniques were used. In this paper, partial feature extraction technique was used, which overcomes the limitation of the other feature extraction techniques and improves the quality of partial feature [10]. The partial feature extraction technique is based on a geometrical invariant function such as sine, cosine, and tangent function and creates shape and size feature [5]. The feature extraction process gives a large amount of feature in terms of lower content feature and higher content of feature. Most of the authors do not consider the lower content of feature. Feature optimization technique was used for the lower content of feature. The feature optimization technique optimized the optimal feature required for the process of feature extraction. In this paper, a swarm intelligence based feature optimization technique is used. The swarm intelligence family consists of various algorithms such as particle swarm optimization, ant colony optimization, and glowworm algorithm. These three algorithms are used for the process of feature optimization. The particle swarm optimization technique is a well-known dynamic population based optimization technique. The concept of particle swarm intelligence is based on bird forks. The bird forks maintain the continuous velocity and speed for flying and define two parameters namely Gbest and Pbest. The ant colony optimization technique works on the nature of biological ants. The biological ants find the path nearest to food. The glowworm optimization algorithm is especially useful for a simultaneous search of multiple optima, usually having different objective function values [9]. To achieve this goal, a swarm must have the ability to split into disjointed groups. Otherwise, only one (local or global) optimum will be found. In GSO, agents exchange information locally. Moreover, their movements are non deterministic. Section II of this paper describes partial feature extraction technique. Section III discusses feature optimization technique. Section IV discusses the experimental result analysis and Section $\mathrm{V}$ is the conclusion.

\section{PARTIAL SHAPE FEATURE EXTRACTION}

This section discusses the feature extraction technique. The feature extraction technique is a very important phase of content-based image retrieval. The partial feature exaction technique has been used for feature extraction. The partial feature extraction technique was derived by Kirti Jain in 2013 [10]. The conventional shape-based feature extraction, such as chain code, edge detection, and Hough transform function are used for outer boundary feature detection. When the shape of the image used is of triangular and trapezoidal pattern the extraction of feature process such as chain code and edge detection suffer. Hence, some authors used ringlet transform function [3] for resolution of point function. However, the computation of point function is very complex, and so is the feature extraction process. The sin function, cosine function and tangent function are used for partial feature extraction based on boundary value of an image. The given image is divided into three sections namely hypotenuse, opposite, and adjacent. These three parameters are ontained before applying the edge detection technique for getting the $\mathrm{X}$ and $\mathrm{Y}$ 
parameter in the plane. For better continuity of edge detection, the canny edge detection is used. The derivation process is now explained as formulae.

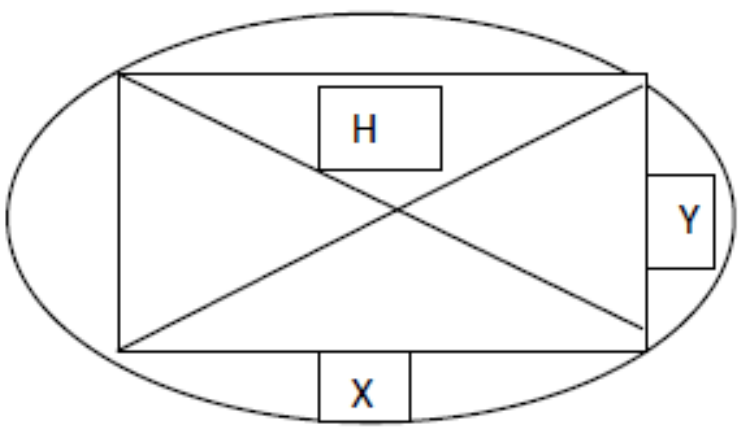

Fig. 1. Circular image divided into three sections: $\mathrm{X}, \mathrm{Y}$ and $\mathrm{H}$

The following steps explain the process of feature extraction using the triangular formula.

1) Apply canny edge detection method for finding the boundary value of the image

2) Find the centred point of boundary value of shape

3) Find the Xc and $Y c$ as

$\mathrm{Xc}=\sum_{i=1}^{n} x i / n$

$\mathrm{Yc}=\sum_{i=1}^{n} y i / n$.

4) After getting a value of (Xc and $Y c$ )

$$
\mathrm{H}=\sqrt{a^{2}+b^{2}} \text { Where } \mathrm{a}=\sum_{i=1}^{n} \frac{x i}{n}, b=\sum_{i=1}^{n} \frac{y i}{n}
$$

5) After getting the value of $H$ apply sine, cosine, and tangent function for shape of the boundary

6) $\operatorname{Sin}=X c / H$ and cosine $=Y c / H$ and tangent $=Y c / X c$

7) After getting sin, cosine, and tangent, find three consecutive matrix of shape

8) All three shape parameters match the boundary value of the feature.

This is the basic principle component of partial shape feature extraction process in image retrieval.

\section{FEATURE OPTIMIZATION PROCESS}

For the optimization of feature, three swarm intelligence algorithms namely particle swarm optimization, ant colony optimization, and glowworm optimization algorithm are used. All these optimization algorithms are used in three different sections. The first section discussed the particle swarm optimization technique in terms of feature optimization; the second section discusses ant colony optimization technique in terms of feature optimization; and the third section discusses the glowworm optimization algorithm for feature optimization.

\section{A. First Section}

The particle swarm optimization feature optimizer selects the input of image feature (partial feature) in terms of $D^{R}$ where $\mathrm{D}$ is the value of domain database and $\mathrm{R}$ shows the feature component of $\mathrm{R}$ image. The $\mathrm{R}$ image feature content $\{r 1, r 2, r 3, r 4, \ldots \ldots . r n\}$ describes the artificial particle as population. The unique feature relation of input image sets the velocity of the particle. If the feature attribute value is changed, then the next iteration moves the update of velocity. These terms describe the particle's feature value; Rid and its near value of a particle; and Rgd which is the velocity value of optimization feature space. The random values for feature are fet 1 and fet 2 which are used for the local and global value selection of particle, that is, to make the optimal solution. The values of c1 and c2 manage the value of velocity of Rid and Rgd in deciding the particle's next movement velocity. Each iteration changes the velocity of swarm and creates a new feature subset for selection of feature. The derivation of equation in (3) and (4) [9]

$$
\begin{gathered}
v_{i d=W \times V_{i d}+C 1 \times f e t 1 \times\left(R_{i d-x_{i d}}\right)+C_{2} \times r a n d 2 \times\left(R_{g d-X_{i d}}\right)} C \ldots \ldots \ldots \\
X_{i d=X_{i d}+V_{i d} \ldots \ldots \ldots \ldots \ldots \ldots \ldots . . .}(4)
\end{gathered}
$$

Where $w$ denotes the value of feature matrix; Rid is the position of particle value, $\mathrm{Rgd}$ is the position of global value best fitness value, c1 and $\mathrm{c} 2$ are constants and are known as acceleration coefficients; $\mathrm{d}$ denotes the dimension of the problem space; and fet 1 , fet 2 are random values in the range of $(0,1)$.

\section{B. Second Section}

The extracted partial feature passes through ant colony optimization. The ant colony optimization process finds the continuity of shape feature. The process of ant colony optimization technique is basically described in terms of artificial ants. This process finds the dissimilar and redundant group of partial features [6]. Te process of feature optimization is described here. The process of feature optimization of partial feature data passes through the feature space of ant colony optimization. The mapping of partial data feature attributes according to their artificial ants requires some standard derivation and parameter. On the basis of the parameters, the feature similarity of two different shapes is estimated. The most similar features pass through the process of retrieval and increase the capacity of precision and recall.

When $\mathrm{F}$ is a feature set and $\mathrm{N}$ is the total artificial ants and possibility of ant selection is $\mathrm{s} 1, \mathrm{~s} 2 \ldots \ldots \ldots \ldots \mathrm{sn}$, the selection possibility of two ants in giving solution is as follows.

$$
S P(i, j)=\frac{1}{s i-s j} .
$$

Where si and sj are the dissimilar probability of two different ants. Now the value of appetence of ants isestimated as

$$
\operatorname{ACP}(\mathrm{i}+\mathrm{j})=\frac{\alpha i+\beta i}{N}
$$

Where $\alpha \mathrm{i}$ and $\beta \mathrm{i}$ are ants whose selection possibility is maximum in terms of other ants; the ratio of selection of ants is defined as $\frac{100}{N}$

On the basis of selection possibility, the value of artificial phenomenon value is estimated as follows.

$$
\Delta \tau i=\frac{A \cdot s i}{A C P(i+j)}
$$

Where $\mathrm{A}$ is a constant phenomenon value 
Now each iteration of pheromone value is increment and decrement according to their selection probability. The derivation of universal appetence probability is

$$
p_{i i}^{k}=\left\{\begin{array}{cc}
{\left[\tau_{i j(t)}\right]} \\
0 \quad \text { otherwise }
\end{array} \quad \alpha \cdot\left[k_{i j}\right] \beta i f j € j \ldots \ldots \ldots(8)\right.
$$

Where kij gives the information of heuristic search space and measures the selection possibility of artificial ants

Finally, we get the optimal partial feature of image database for the purpose of optimization.

\section{Third Section}

The extracted partial feature passes through the glowworm algorithm. The partial feature is mapped into the glowworm search space. Each glowworm encodes the object function value $J(x i(t))$ at its current location $x i(t)$ into $\alpha$ luciferin value li and broadcasts the same within its neighborhood. The set of neighbor $(\mathrm{Ni}(\mathrm{t}))$ of glowworm i consists of those glowworms that have a relatively higher luciferin value located within a dynamic decision domain and updating by formula 4.1 at each iteration[11].

Local decision range update is given by equation " 9 "

$$
\begin{gathered}
r_{d}^{i}(t+1)=\min \left\{r s, \max \left\{0, r_{d}^{i}(t)+\beta(n t\right.\right. \\
-|N i(t)|)\}\} \ldots \ldots \ldots \ldots \ldots \ldots \ldots \ldots \ldots \ldots \ldots \ldots \ldots \ldots \ldots
\end{gathered}
$$

And $r_{d}^{i}(t+1)$ is the glowworm is local decision range at the $t+1$ iteration, $r s$ is the sensor range, and $n t$ is the neighborhood range. The number of glow in local decision range is given by equation " 10 "

$$
N_{i(t)}=\left\{j:\|x i(t)-x i(t)\|<r_{d}^{i} ; l i(t)<l i(t)\right\} \ldots
$$

$\mathrm{xi}(\mathrm{t})$ is the glowworm I position at the titeration; $(\mathrm{t})$ is the glowworm luciferin at the $t$ iteration. The set of neighbors of glowworm consists of those glowworms that have relatively higher luciferin value and are located within the dynamic decision domain whose range $r_{d}^{i}$ is bounded above by a circular sensor range.

Each glowworm is given in equation " 11 "

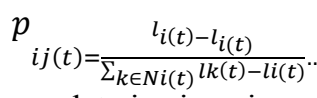

Movement update is given in equation " 12 "

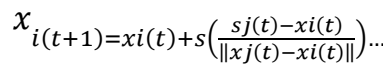

Luciferin update is given in equation " 13 "

$$
l_{i(t)=(1-\rho) l i(t-1)+\gamma j(x i(t))} \text {. }
$$

And $\operatorname{li}(\mathrm{t})$ is a luciferin value of glowworm $\mathrm{i}$ at the $\mathrm{t}$ iteration, $\mathrm{P}$ belong $(0,1)$ lead to the reflection of the cumulative kindness of the path followed by the glowworm in their current luciferin values the parameter $\mathrm{Y}$ only scale the function values, $J(x i(t))$ is the value of test function. Finally, the optimal feature is obtained. The optimal feature of image database passes through the image retrieval process.

\section{RESUlt ANALYSIS OF IMAGE RETRIEVAL}

This section discusses the performance evaluation of three swarm intelligence algorithms for content-based image retrieval. For the evaluation of performance, coral image dataset 10000 was used. This coral image dataset was divided into different dataset models in terms of 1000, 2000, 5000, and 10000 and MatLab software was used for implementing the swarm-based algorithm. MatLab is a well-known computational algorithmic software. For the evaluation of performance, precision and recall were used as the two parameters. For the calculation of precision and recall, the following parameters were used.

TP- True positive

TN True Negative

FP False Positive

FN False Negative

$$
\begin{aligned}
& \text { Pricision }=\frac{T P}{(T P+F P)} \ldots \ldots \ldots \ldots \ldots \ldots \ldots \ldots(a) \\
& \text { Recall }=\frac{T P}{(T P+F N)} \ldots \ldots \ldots \ldots \ldots \ldots \ldots \ldots \ldots(b)
\end{aligned}
$$

Formula (a) and (b) measure the performance of precision and recall during the process of experimental evaluation.

TABLE I. COMPARATIVE PRECISION AND RECALl OF IMAGE RETRIEVAL BASED CLASSIFICATION FOR A TOTAL OF 1000 IMAGES

\begin{tabular}{|l|l|l|l|}
\hline Data set & Method & Precision $(\%)$ & Recall (\%) \\
\hline \multirow{4}{*}{ Car images } & GSO & 88.66 & 82.22 \\
\cline { 2 - 4 } & ANT & 89.33 & 85 \\
\cline { 2 - 4 } & PSO & 91.26 & 87 \\
\hline \multirow{4}{*}{ Bird images } & GSO & 91.26 & 93.05 \\
\cline { 2 - 4 } & ANT & 92.48 & 94.06 \\
\cline { 2 - 4 } & PSO & 94.58 & 95.68 \\
\hline \multirow{3}{*}{$\begin{array}{l}\text { Cartoon images } \\
\text { images }\end{array}$} & GSO & 86.33 & 80.81 \\
\cline { 2 - 4 } & ANT & 88.79 & 81.34 \\
\cline { 2 - 4 } & PSO & 90.76 & 83.67 \\
\hline \multirow{3}{*}{$\begin{array}{l}\text { Multiple bird } \\
\text { images }\end{array}$} & GSO & 85.33 & 78.83 \\
\cline { 2 - 4 } & ANT & 91.33 & 81.33 \\
\cline { 2 - 4 } & GSO & 93.67 & 85.89 \\
\cline { 2 - 4 } & ANT & 88.66 & 89.66 \\
\cline { 2 - 4 } & PSO & 92.49 & 80.68 \\
\hline
\end{tabular}


TABLE II. COMPARATIVE PRECISION AND RECALL OF IMAGE RETRIEVAL BASED CLASSIFICATION FOR A TOTAL OF 2000IMAGES

\begin{tabular}{|l|l|l|l|}
\hline Data set & Method & Precision (\%) & Recall (\%) \\
\hline \multirow{3}{*}{ Car images } & GSO & 85.89 & 82.22 \\
\cline { 2 - 4 } & ANT & 87.33 & 84.67 \\
\cline { 2 - 4 } & PSO & 93.56 & 88.79 \\
\hline \multirow{3}{*}{ Bird images } & GSO & 89.78 & 91.35 \\
\cline { 2 - 4 } & ANT & 93.88 & 92.16 \\
\cline { 2 - 4 } & PSO & 94.64 & 94.68 \\
\hline \multirow{3}{*}{ Cartoon images images } & GSO & 87.46 & 81.76 \\
\cline { 2 - 4 } & ANT & 90.49 & 83.44 \\
\cline { 2 - 4 } & PSO & 92.86 & 85.72 \\
\cline { 2 - 4 } & GSO & 88.43 & 83.23 \\
\cline { 2 - 4 } & ANT & 92.63 & 89.79 \\
\hline \multirow{2}{*}{$\begin{array}{l}\text { Multiple bird } \\
\text { images }\end{array}$} & GSO & 95.22 & 82.36 \\
\cline { 2 - 4 } & ANT & 89.36 & \\
\cline { 2 - 4 } & PSO & 95.46 & \\
\hline
\end{tabular}

TABLE III. COMPARATIVE PRECISION AND RECALl OF IMAGE RETRIEVAL BASED CLASSIFICATION FOR A TOTAL OF 5000 IMAGES

\begin{tabular}{|c|c|c|c|}
\hline Data set & Method & Precision (\%) & Recall (\%) \\
\hline \multirow{3}{*}{ Car images } & GSO & 86.97 & 84.78 \\
\hline & ANT & 89.43 & 85.7 \\
\hline & PSO & 94.52 & 91.46 \\
\hline \multirow{3}{*}{ Bird images } & GSO & 90.48 & 92.5 \\
\hline & ANT & 92.86 & 93.6 \\
\hline & PSO & 95.66 & 94.66 \\
\hline \multirow{3}{*}{ House images } & GSO & 89.47 & 83.79 \\
\hline & ANT & 91.89 & 85.54 \\
\hline & PSO & 93.46 & 87.78 \\
\hline \multirow{3}{*}{ Cartoon images } & GSO & 89.43 & 82.23 \\
\hline & ANT & 91.69 & 85.7 \\
\hline & PSO & 93.62 & 89.79 \\
\hline \multirow{3}{*}{$\begin{array}{l}\text { Multiple bird } \\
\text { images }\end{array}$} & GSO & 91.65 & 80.86 \\
\hline & ANT & 93.4 & 82.44 \\
\hline & PSO & 95.7 & 84.5 \\
\hline
\end{tabular}

TABLE IV. COMPARATIVE PRECISION AND RECALl OF IMAGE RETRIEVAL BASED CLASSIFICATION FOR A TOTAL OF 10000 IMAGES

\begin{tabular}{|l|l|l|l|}
\hline Data set & Method & Precision (\%) & Recall (\%) \\
\hline \multirow{3}{*}{ Car images } & GSO & 89.47 & 88.6 \\
\cline { 2 - 4 } & ANT & 91.63 & 89.4 \\
\cline { 2 - 4 } & PSO & 94.02 & 93.66 \\
\hline \multirow{3}{*}{ Bird images } & GSO & 91.48 & 93.5 \\
\cline { 2 - 4 } & ANT & 92.76 & 94.62 \\
\cline { 2 - 4 } & PSO & 95.48 & 95.7 \\
\hline \multirow{3}{*}{ Cartoon images } & GSO & 91.7 & 83.86 \\
\cline { 2 - 4 } & ANT & 93.8 & 85.5 \\
\cline { 2 - 4 } & PSO & 95.56 & 87.7 \\
\hline \multirow{3}{*}{$\begin{array}{l}\text { Multiple bird } \\
\text { images }\end{array}$} & GSO & 91.3 & 82.28 \\
\cline { 2 - 4 } & ANT & 93.6 & 85.76 \\
\cline { 2 - 4 } & PSO & 95.68 & 89.8 \\
\cline { 2 - 4 } & GSO & 93.69 & 84.23 \\
\cline { 2 - 4 } & PSO & 95.46 & \\
\hline
\end{tabular}

\section{Comparative result of 1000 images using GSO, ANT and PSO method}

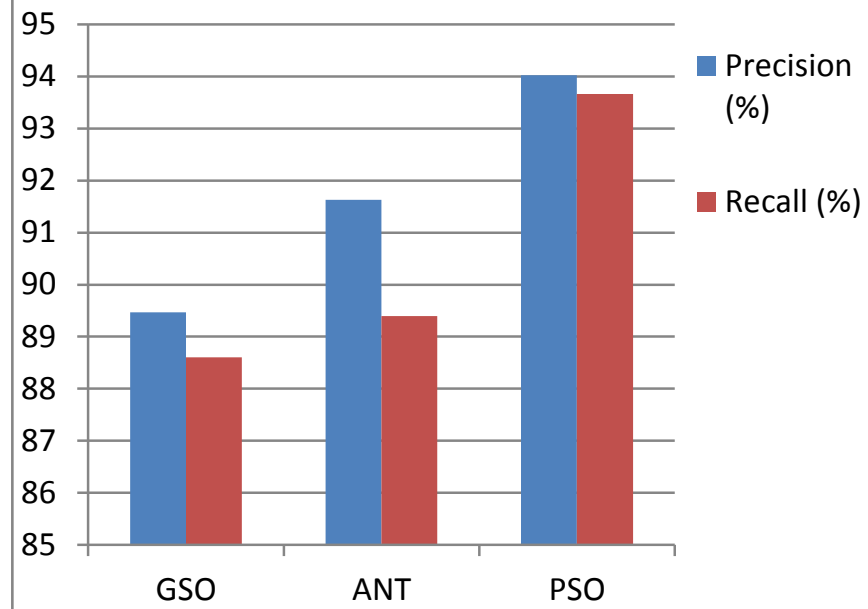

Fig. 2. Shows performance of a data set for 1000 image counts of data and rate of precision and recall on the base of GSO, ANT and PSO method. The above graph shows the comparative result analysis of image data sets of 1000 images with three different methods and calculates the parameter value of precision and recall. The variation of result in precision and recall on the basis of applying three methods found that the result of PSO is better than GSO and ANT

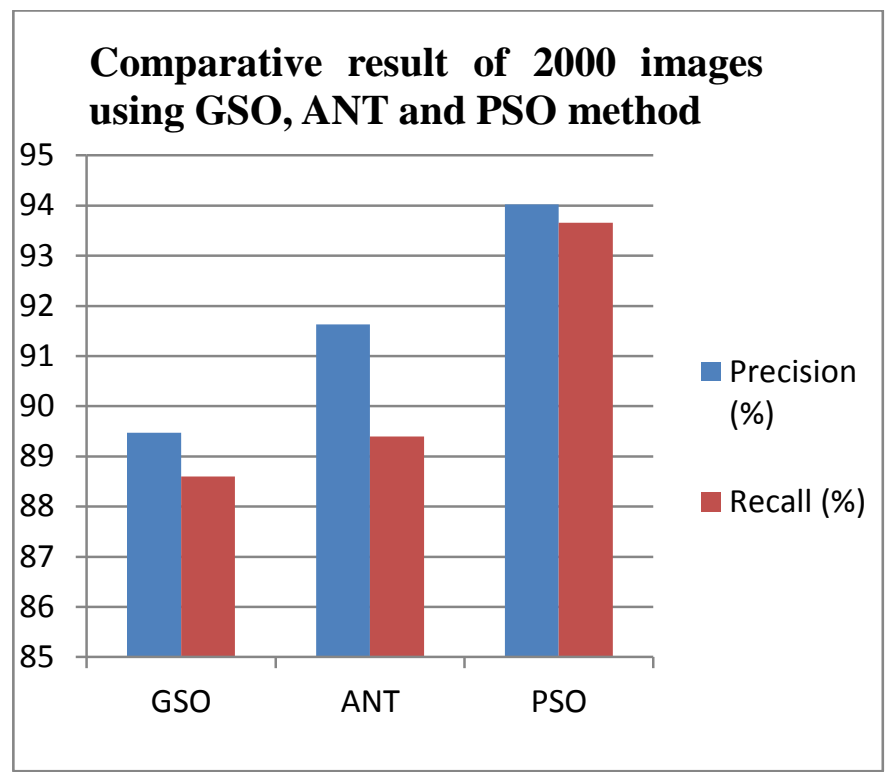

Fig. 3. Shows performance of a data set for 2000 image counts of data and rate of precision and recall on the base of GSO, ANT and PSO method. The above graph shows the comparative result analysis of image data sets of 2000 images with three different methods and calculates the parameter value of precision and recall. The variation of results in precision and recall on the basis of applying three methods found that the result of PSO is better than GSO and ANT 


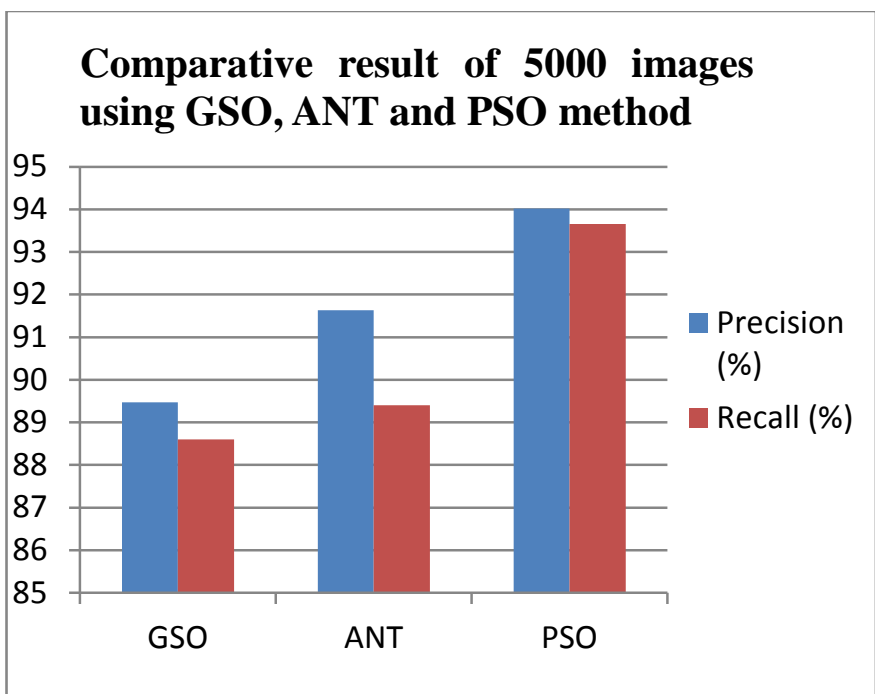

Fig. 4. Shows performance of data set for 5000 image counts of data and rate of precision and recall on the base of GSO, ANT and PSO method. The above graph shows the comparative result analysis of image data sets of 5000 images with three different methods and calculates the parameter value of precision and recall. The variation of result in precision and recall on the basis of applying three methods found that the result of PSO is better than GSO and ANT

\section{Comparative result of 10000 images using GSO, ANT and PSO method}

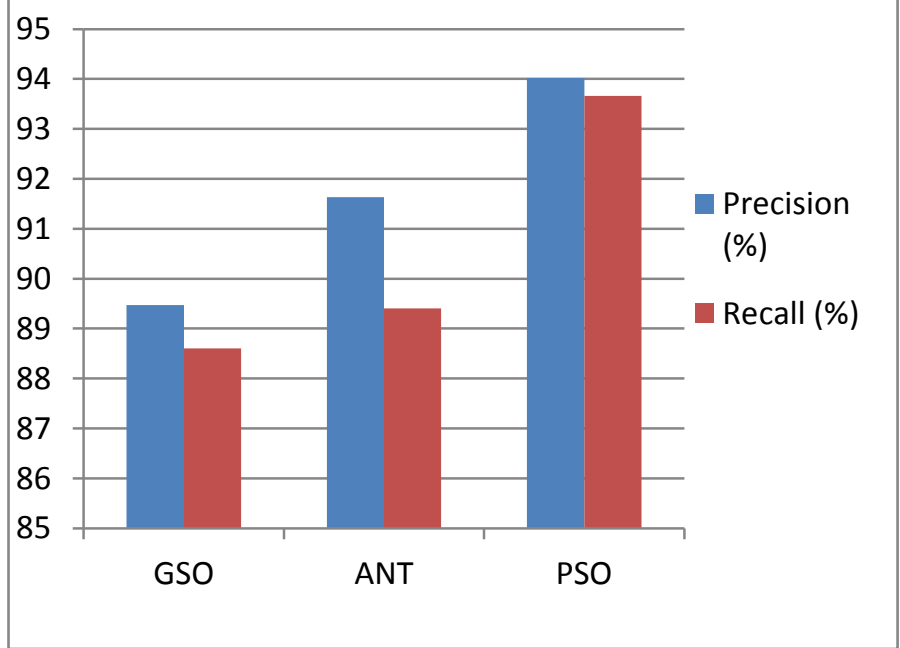

Fig. 5. Shows performance of a data set for 10000 image counts of data and rate of precision and recall on the base of GSO, ANT and PSO method. The above graph shows the comparative result analysis of image data sets of 10000 images with three different methods and calculates the parameter value of precision and recall. The variation of results in precision and recall on the basis of applying three methods found that the result of PSO is better than GSO and ANT

\section{CONCLUSION AND FUTURE WORK}

This paper presents the performance evaluation of feature optimization based content-based image retrieval. In contentbased image retrieval, the partial feature extraction technique is used. These partial feature extraction techniques are very efficient methods for feature extraction. For the optimization of feature, three algorithms namely ACO, GSO, and PSO were used. The diversity of particle swarm optimization is very high and due to this the optimization ,performance is better than glowworm and ACO algorithm. Our empirical evaluation of results shows that particle swarm optimization has better precision and recall value and is a bit different than ACO and glow worm algorithm. The ACO faced problem with feature discontinuity and does not get satisfactory results. The glowworm algorithm uses limited set of features and produces better results, but all features and their sub-sets suffered a problem of precision and recall of content-based image retrieval. In future design, feature-based fitness constraints function for ACO and glowworm algorithm will be addressed.

\section{REFERENCES}

[1] Liang-Hua Chen, Yao-Ling Hung, Li-Yun Wang (2012) An Integrated Approach to Image Retrieval. IEEE, .Pp 695-700.

[2] Dengsheng Zhang, GuojunLu (2004) Review of shape representation and description techniques. Elsevier, Pattern Recognition 37 Pp1-19.

[3] Minh N. Do, MartinVetterli, (2003) The Finite Ridgelet Transform for Image Representation. IEEE TRANSACTIONS ON IMAGE PROCESSING, VOL. 12.

[4] EsmatRashedi, HosseinNezamabadi-pour (2012)Improving the Precision of CBIR Systems by Feature Selection Using Binary Gravitational Search Algorithm.The 16th CSI International Symposium on Artificial Intelligence and Signal Processing, IEEE Pp 39-43.

[5] Y.L. Wu, C.Y. Tang, M.K. Hor, P.F. Wu (2011)Feature selection using genetic algorithm and cluster validation Expert Systems with Applications, Vol-38, Pp 2727-2732.

[6] V.SelviDr.R.Umarani (2010) Comparative Analysis of Ant Colony and Particle Swarm Optimization Techniques International Journal of Computer Applications Volume 5- No.4.

[7] MattiaBroilo, Francesco G. B. De Natale (2010) A Stochastic Approach to Image Retrieval Using Relevance Feedback and Particle Swarm Optimization, IEEE TRANSACTIONS ON MULTIMEDIA, VOL12,No.4Pp 267-277.

[8] Ayesha Salahuddin, AlinaNaqvi, KainatMujtaba, JunaidAkhtar (2012) Content based Video Retrieval using Particle Swarm Optimization 10th International Conference on Frontiers of Information Technology, IEEE, Pp 79-83.

[9] S.W. Lin, K.C. Ying, S. Chen, Z.J. Lee (2008) Particle swarm optimization for parameter determination and feature selection of support vector machines Expert systems with applications" Elsevier, Pp 1817-1824.

[10] Kirti Jain, Dr.Sarita Singh and Dr.Gulab Singh" Partial Feature Based Ensemble of Support Vector Machine for Content based Image Retrieval" in International Journal of Innovative Research in Computer and Communication Engineering 2013, Pp622-625.

[11] TaoChen ,Huifang Deng and Chunru Dong" An Image Retrieval Method Based on Multi-instance Learning and PerturbativeGlowworm Swarm Optimization" in Journal of Information \& Computational Science July 2013. 